
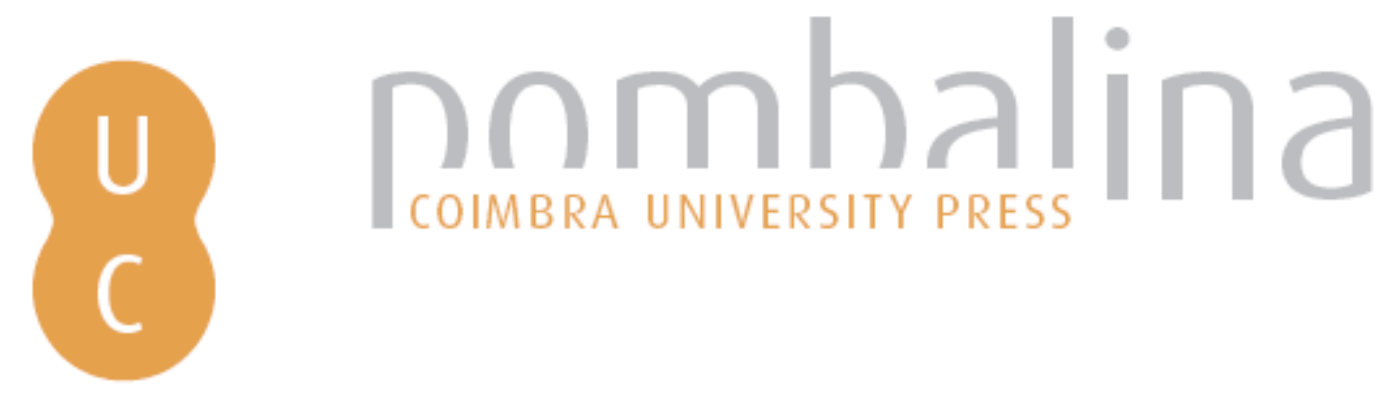

\title{
Sistema de apoio à decisão e espacial para análise do impacte ambiental da dispersão de poluentes atmosféricos
}
Autor(es):
Alçada-Almeida, Luís; Coutinho-Rodrigues, João; Sousa, Nuno
Publicado por: Imprensa da Universidade de Coimbra
URL
persistente:
URI:http://hdl.handle.net/10316.2/37056
DOI:
DOI:http://dx.doi.org/10.14195/978-989-26-0983-6_3
Accessed : $\quad$ 26-Apr-2023 15:42:02

A navegação consulta e descarregamento dos títulos inseridos nas Bibliotecas Digitais UC Digitalis, UC Pombalina e UC Impactum, pressupõem a aceitação plena e sem reservas dos Termos e Condições de Uso destas Bibliotecas Digitais, disponíveis em https://digitalis.uc.pt/pt-pt/termos.

Conforme exposto nos referidos Termos e Condições de Uso, o descarregamento de títulos de acesso restrito requer uma licença válida de autorização devendo o utilizador aceder ao(s) documento(s) a partir de um endereço de IP da instituição detentora da supramencionada licença.

Ao utilizador é apenas permitido o descarregamento para uso pessoal, pelo que o emprego do(s) título(s) descarregado(s) para outro fim, designadamente comercial, carece de autorização do respetivo autor ou editor da obra.

Na medida em que todas as obras da UC Digitalis se encontram protegidas pelo Código do Direito de Autor e Direitos Conexos e demais legislação aplicável, toda a cópia, parcial ou total, deste documento, nos casos em que é legalmente admitida, deverá conter ou fazer-se acompanhar por este aviso. 


\section{$\forall$ \\ TAS DAS I JORNADAS LUSÓFONAS DE CIÊNCIAS E TECNOLOGIAS DE INFORMAÇÃO GEOGRÁFICA}

Editores

José Gomes dos Santos

Cidália Fonte

Rui Ferreira de Figueiredo

Alberto Cardoso

Gil Gonçalves

José Paulo Almeida

Sara Baptista 


\section{ARTIGO 3}

\section{SISTEMA DE APOIO À DECISÃO ESPACIAL PARA ANÁLISE DO IMPACTE AMBIENTAL DA DISPER- SÃO DE POLUENTES ATMOSFÉRICOS}

ALÇADA-ALMEIDA, Luis ${ }^{1,2}$; COUTINHO-RODRIGUES, João $0^{1,3}$ E SOUSA, Nuno ${ }^{1,4}$

\footnotetext{
${ }^{1}$ Instituto de Engenharia de Sistemas e Computadores (INESC-Coimbra); Rua Antero Quental 199, 3000033 Coimbra, Portugal; Tel: +351 239 851040/9; Fax: +351 239824692.

2 Faculdade de Economia da Universidade de Coimbra; Avenida Dias da Silva 165, 3004-512 Coimbra, Portugal; Tel: +351 239790 558; Fax: +351 239790 514; email: alcada@dec.uc.pt

3 Departamento de Engenharia Civil da Faculdade de Ciências e Tecnologia da Universidade de Coimbra; Rua Luís Reis Santos - Pólo II, 3030-788 Coimbra, Portugal; Tel: +351 239 797145; Fax: +351 239 797123; email: coutinho@dec.uc.pt

${ }^{4}$ Departamento de Ciências e Tecnologia da Universidade Aberta; Delegação de Coimbra da Universidade Aberta; Rua Alexandre Herculano, n. ${ }^{\circ}$ 52, 3000-019 Coimbra, Portugal; Tel: +351 300 001590; Fax: +351300001599; email: nsousa@uab.pt
}

\section{RESUMO}

Neste artigo é apresentado um Sistema de Apoio à Decisão Espacial (SADE) onde os decisores podem facilmente definir diferentes tipos de problemas espaciais recorrendo a diferentes categorias de objetos, pré-definidas ou a definir, associando- lhes características com ou sem dependência espacial, e indicando formas de interferência (impactos) entre essas características/propriedades. A análise espacial para determinação ou avaliação de configurações alternativas para a localização de diferentes tipos de ocorrências espaciais será feita através da utilização interativa do SADE de acordo com conjuntos de regras intrínsecas aos vários elementos gráficos (objetos, categorias, características, impactos) utilizados na definição dos problemas. O teste à generalidade representativa e analítica do SADE proposto é efec- 
tuado recorrendo a um problema concreto, suficientemente específico e complexo, relativo à aplicação de modelos gaussianos para o estudo da dispersão atmosférica de eventuais poluentes resultantes do tratamento de resíduos sólidos. A região em estudo está limitada, neste exemplo, ao município de Coimbra, Portugal. Para este município estão acessíveis, e são utilizados, os dados demográficos ao nível da secção de voto (censos oficiais) e, como tal, é possível a realização de um estudo realístico do impacto com populações humanas.

\title{
PALAVRAS-CHAVE
}

Sistemas de informação geográfica (SIG), Modelação de problemas, Modelos gaussianos de dispersão atmosférica, Impacto ambiental, Interface com decisores.

\section{A SPATIAL DECISION SUPPORT SYSTEM FOR ENVIRONMENTAL IMPACT ASSESSMENT OF ATMOSPHERIC POLLUTANTS DISPERSION}

\begin{abstract}
In this work a Spatial Decision Support System (SDSS) is presented, where decision makers can easily define different types of spatial problems, resorting to different object categories, pre-defined or to be defined, associating to them space dependent and non-dependent charecterisitics, and indicating forms of interference (impact) between those characteristics/ properties. The spatial analysis for determining or evaluating alternative configurations for the location of different types of spatial occurrences is done interactively through the SDSS, according to sets of rules intrinsic to the various graphical elements (objects, categories, characteristics, impacts) used in the problems definitions. The representative and analytical generality of the SDSS is tested in a case study, which is specific and complex enough, relating to the application of gaussian air dispersion models to pollutants resulting from solid waste treatment. The study region of the case study is the municipality of Coimbra, Portugal. For this municipality, demographical data the ward voting section level (official census data) were available and used. As such, a realistic study of the impact on human populations is possible.
\end{abstract}

\section{KEYWORDS}

Geographical information systems (GIS), Modelling, Gaussian air dispersion models, Environmental impact assessment, Interface with decision makers. 


\section{INTRODUÇÃO}

Os avanços tecnológicos na área da informática, especialmente nas vertentes que mais podem contribuir no âmbito dos SIG (hardware e software de aquisição, visualização e impressão de informação espacial, software de gestão de bases de dados, desenvolvimento dos interfaces gráficos, etc.), possibilitaram-lhes uma viragem radical no sentido de apoiar, na prática, uma grande diversidade de atividades científicas, comerciais e administrativas (Gestão Ambiental, Engenharia Urbana, Meteorologia, Botânica, Zoologia, Matemática, Geografia, Geologia, Planeamento Territorial, Marketing, etc.). Esta evolução não tem sido acompanhada, na sua especificidade, por uma evolução correspondente na área dos Sistemas de Apoio à Decisão (SAD). Neste ponto não estamos a falar de SAD que consultem uma base de dados e que apresentem os seus resultados graficamente servindo-se de um SIG. Este é um triângulo funcional básico, usado como modelo por muitos autores (JANKOWSKI et al., 1997; ALMEIDA \& COUTINHO-RODRIGUES, 2002, 2011; MALCZEWSKI et al., 1999; NATIVIDADE-JESUS et al., 2007), que não implica nenhuma evolução significativa nas três componentes. A evolução que se pretende apresentar neste trabalho pode ser condensada nos seguintes três aspectos:

$\mathrm{Na}$ possibilidade dos SAD (e dos métodos neles incluídos) fugirem à estrutura rígida que em geral limita o tipo de problemas a analisar. Assim, o mesmo sistema pode representar e analisar, de forma integrada, diferentes tipos de problemas que apenas têm em comum uma forte componente espacial, com todas as vantagens para os atuais problemas reais de decisão que são tipicamente evolutivos e sofrem interdependências.

$\mathrm{Na}$ adequação dos SAD ao tratamento de uma intrínseca característica espacial adaptável às diferentes necessidades representativas e analíticas. Esta característica espacial não se esgota na visualização geográfica das soluções: relaciona-se essencialmente com o facto de todo o processo de decisão ser condicionado pelas localizações espaciais dos objetos (dentro do universo multidimensional limitado, definido para cada problema) e pelas suas restantes características de teor espacial ou não espacial, encontradas na estrutura de dados associada. Como exemplo, ao longo de um processo de decisão sobre escolha de trajetos para uma rede de dis- 
tribuição de serviços, a qualidade do pavimento associado aos percursos pode, a dada altura, surgir como um aspecto importante. No entanto, essa característica varia ao longo dos próprios percursos e o sistema tem que fornecer soluções para a representação e inclusão dessa particularidade nos modelos de decisão.

$\mathrm{Na}$ normalização do funcionamento conjunto das três vertentes como um todo (estrutura de dados relacional, apoio à decisão e teor espacial da informação) e apelidar esse conjunto de Sistema de Apoio à Decisão Espacial (SADE), com todas as vantagens reconhecidas de integrar, num mesmo sistema, estruturas flexíveis de representação de informação mono-dimensional e espacial, software SIG e processos analíticos (TURBAN et al., 2010).

Neste trabalho, em vez de se fundamentar toda a concepção do sistema na resolução de um problema específico, pretende-se construir uma plataforma de trabalho genérica que permita, não só analisar problemas espaciais de diversos tipos, mas ainda construir esses problemas de raiz, de acordo com um conjunto de regras de representação e associação de componentes. Deste modo, a definição do problema espacial fica completamente estabelecida através de um conjunto mais ou menos extenso de ações intuitivas no interface gráfico. O agente de decisão (AD) utiliza um sistema de edição, do tipo manipulação direta, onde vai colocando objetos de diferentes categorias, descrevendo a variação das suas características, indicando impactos entre estas, e observando os resultados gráficos produzidos por métodos genéricos de análise espacial de impactos. A definição completa do problema de decisão espacial fica assim ao alcance de um qualquer AD que tenha alguma experiência em sistemas operativos gráficos.

O caso de estudo apresentado é exemplificativo destas funcionalidades, que são genéricas, no sentido em que nada no sistema foi implementado a pensar especificamente em chaminés ou dispersão de poluentes (c.f. abaixo para detalhes). Neste caso de estudo tudo foi criado de raiz pelo utilizador através de operações de desenho e definição de funções. O utilizador podia ter criado p.ex. estradas em vez de chaminés, com descrição dinâmica ou estática do respetivo tráfego, e definido funções de 
dispersão de poluentes ou outros efeitos (p.ex. ruído) e assim estudar o impacto de uma fonte não-pontual destes sobre populações ou outras entidades.

\section{O PROBLEMA}

Os problemas ambientais causados por fontes poluidoras pontuais (exemplo: chaminés) ou lineares (exemplo: vias de comunicação) têm assumido uma importância crescente devido à também crescente concentração das populações em áreas cada vez mais circunscritas, ao crescimento generalizado dos níveis de poluição e a uma consciencialização crescente da própria sociedade para este tipo de problemas (CORBURN, 2007). Assim, tem crescido a necessidade de desenvolver adequados métodos científicos para estudar os efeitos de possíveis acidentes, descrevendo, e antecipando com rigor, a forma como os possíveis poluentes se iriam distribuir e afectar as diferentes populações animais e vegetais, possibilitando fases de planeamento que tenham em conta a diminuição dos riscos envolvidos. Diferentes modelos têm sido construídos para representar estes fenómenos, quer se trate da propagação livre (exemplo: pelo ar) ou restringida a redes (exemplo: cursos de água), de partículas sólidas, gases ou mesmo ruído.

No caso particular da dispersão atmosférica de poluentes de origem pontual, o modelo gaussiano (MAHONEY, 1974; GORDON, 1985; BOUBEL et al., 1994; HOLMES \& MORAWSKA, 2006), assume uma grande relevância pois permite prever a distribuição final das concentrações, em regime "permanente", de acordo com diferentes factores como sejam: o vento e outras características atmosféricas exteriores ao ponto de emissão, características da própria emissão e do canal de saída, tipificação da região envolvente, etc. Devido a estas características, é o modelo mais usado para estimar o impacto de poluentes não reativos sobre territórios e populações (USEPA, 1986, 2003; KIELY, 1997).

Diferentes autores têm implementado sistemas baseados em SIG para modelar este fenómeno particular (ZHANG et al., 2000; ALMEIDA \& COUTINHO-RODRIGUES, 2011). Nesses sistemas, apenas a utilização 
do modelo estaria ao alcance do utilizador final. Todas as fases da sua construção, bem como da adaptação do modelo a diferentes cenários (por exemplo ao estudo do impacto alternativo da abertura de $\mathrm{n}$ pontos de eventual emissão diferenciados e de menor fluxo/risco), seriam da responsabilidade do programador que, quando muito, seguiria orientações do AD.

\section{METODOLOGIA}

A utilização concreta de um SADE com as particularidades lógicas e funcionais descritas, no âmbito do estudo da dispersão de poluentes atmosféricos, passa por definir os objetos representantes das possíveis fontes de emissão de poluentes (chaminés), suas características pontuais de emissão relevantes de forma genérica (cota da saída de poluentes, fluxos, raio de abertura, etc.) e as características zonais influentes (demográficas, atmosféricas, etc.).

\subsection{O Modelo Gaussiano de Dispersão Atmosférica}

A expressão do modelo gaussiano que calcula as concentrações de poluentes em qualquer ponto de coordenadas (x1, y1) (BOUBEL et al., 1994) considera que o eixo dos $x x$ é paralelo à direcção do vento $\mathrm{e}$ que a fonte emissora se situa na origem das coordenadas. Para adaptar esta expressão à lógica do SADE proposto, será necessário generalizar esta expressão para quaisquer origens (s - source) situadas em ponto genéricos (xs, ys ) e tornar a mesma expressão dependente de dois afastamentos de qualquer ponto $(\mathrm{x} 1, \mathrm{y} 1)$ a essas origens: o afastamento $D w$ segundo a direcção do vento ( $w$ - wind) e o afastamento $D p$ segundo uma perpendicular à mesma direcção. Na Figura 1 são representadas esquematicamente estas distâncias e na equação 1 são apresentadas as expressões correspondentes. 


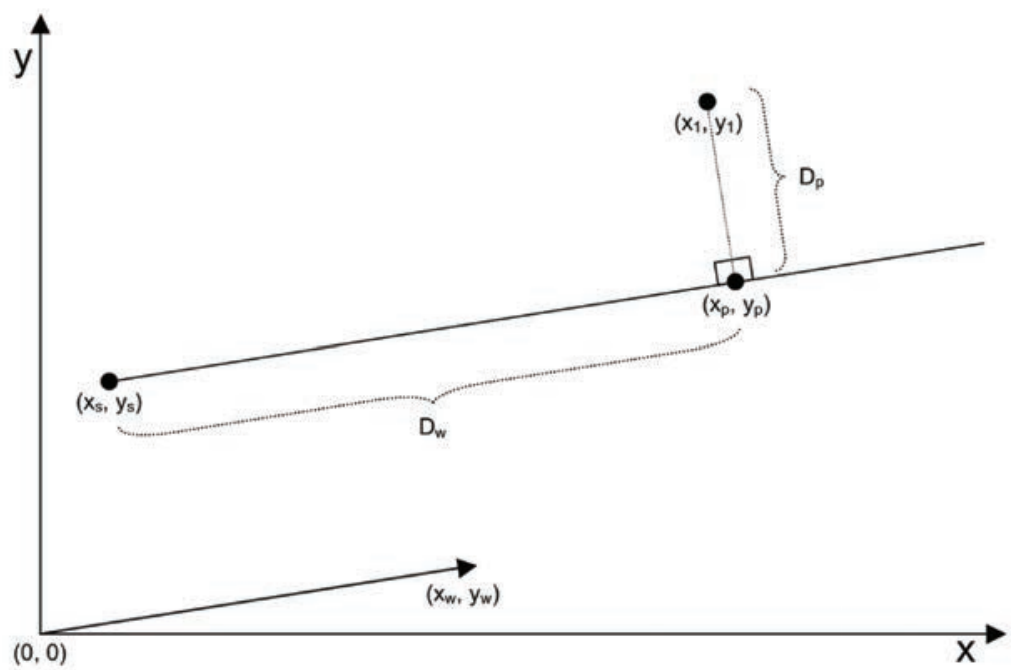

Figura 1 - Distâncias, na direcção do vento $(D w)$ e perpendicular $(D p)$, entre um ponto receptor 1 e uma origem $s$

$$
\left\{\begin{array}{l}
D_{w}=\frac{x_{w}\left(x_{1}-x_{s}\right)+y_{w}\left(y_{1}-y_{s}\right)}{\sqrt{x_{w}^{2}+y_{w}^{2}}} \\
D_{p}=\frac{x_{w}\left(x_{s}-x_{1}\right)+y_{w}\left(y_{s}-y_{1}\right)}{\sqrt{x_{w}^{2}+y_{w}^{2}}}
\end{array}\right.
$$

Eq. 1a. Distância entre um qualquer ponto recetor $\left(x_{1}, y_{1}\right)$ e um ponto origem $\left(x_{s}, y_{s}\right)$ segundo uma direção dada pelo vetor vento $\left(x_{w}, y_{w}\right)$. Valores positivos no sentido do vento.

Eq. 1b. Distância, na perpendicular ao vento, entre um recetor $\left(x_{1}, y_{1}\right)$ e uma origem $\left(x_{s}, y_{s}\right)$.

Numa fase seguinte torna-se necessário alterar a expressão inicial do modelo gaussiano de modo a que a mesma devolva valores nulos em regiões afastadas da origem no sentido contrário ao do vento. Esta generalização torna-se necessária pois as expressões serão aplicadas tanto em regiões sob influência de mais do que uma origem de poluentes, como em regiões afectadas apenas por uma origem ou, eventualmente, livres de qualquer poluição atmosférica (segundo os resultados deste modelo). Dado que o valor de $x^{\prime}$ é negativo nas regiões situadas 'contra o vento', basta adicionar à expressão inicial o módulo desta distância e dividir por dois para anular toda a expressão na região não afectada pelas emissões e mantê-la inalterada na restante área. A expressão final a integrar no 
SADE é a que está presente na equação 2 .

$$
C\left(D_{w}, D_{p}, z\right)=\frac{Q\left(D_{w}+\left|D_{w}\right|\right)}{4 \pi D_{w} \sigma_{y} \sigma_{z} U} e^{-\frac{1}{2}\left(\frac{D_{p}}{\sigma_{y}}\right)^{2}}\left\{e^{-\frac{1}{2}\left(\frac{z-z_{s}}{\sigma_{z}}\right)^{2}}+e^{-\frac{1}{2}\left(\frac{z+z_{s}}{\sigma_{z}}\right)^{2}}\right\}
$$

Eq. 2. Expressão de cálculo da concentração de poluentes num ponto (x,y,z)

$\boldsymbol{C}$ : concentração $C$ em $\left(\boldsymbol{D}_{w}, \boldsymbol{D}_{p}, \mathbf{z}\right)$ da mesma propriedade e nas mesmas unidades de $\boldsymbol{Q}$, por unidade de volume.

$\boldsymbol{x}_{\boldsymbol{w}}, \boldsymbol{y}_{\boldsymbol{w}}$ : componentes $\boldsymbol{x}, \boldsymbol{y}$ do vector velocidade do vento $[\mathrm{m} / \mathrm{s}]$.

$U=\sqrt{x_{w}^{2}+y_{w}^{2}}$ : módulo da velocidade do vento $[\mathrm{m} / \mathrm{s}]$.

$Q$ : emissão de $Q$ unidades por unidade de tempo.

$\sigma_{y}, \sigma_{z}:$ parâmetros de dispersão de acordo com o tipo de zona, classe de estabilidade atmosférica

(Pasquill type) e distância $\boldsymbol{D}_{\boldsymbol{w}}$ ao ponto de emissão $\left(\boldsymbol{x}_{\boldsymbol{s}}, \boldsymbol{y}_{s}\right)$.

$D_{p}$ : distância perpendicular ao vento no ponto de emissão $\left(\boldsymbol{x}_{s}, \boldsymbol{y}_{s}\right)$.

$z_{s}$ : altura efectiva de emissão (altura da chaminé + sobreelevação da coluna de poluentes.

Para que o modelo seja realista torna-se ainda necessário entrar em conta com a sobrelevação da coluna de poluentes acima da altura da chaminé. As várias expressões para representar este fenómeno foram revistas em trabalhos de Briggs (BRIGGS, 1969), mantendo-se como um dos modelos mais utilizados na determinação da altura efetiva de emissão $(\Delta H)$, a fórmula de Oak Ridge desenvolvida pelo US Weather Bureau em 1953. Esta fórmula é apresentada na equação 3 e corresponde ao desenvolvimento do segundo termo da parcela $Z s$ referida na equação 2 .

$$
\Delta H=\frac{2 V_{s} r_{s}}{U}\left[1.5+\left(2.68 \times 10^{2}\right) P\left(1-\frac{T_{a}}{T_{s}}\right) 2 r_{s}\right]
$$

Eq. 3. Elevação adicional da coluna de poluentes à saída da chaminé.

$V_{s}$ : velocidade de emissão na boca da chaminé $[\mathrm{m} / \mathrm{s}]$.

$r_{s}$ : raio da boca da chaminé $[\mathrm{m}]$.

$P$ : pressão atmosférica $[\mathrm{kPa}]$.

$T_{s}$ : temperatura da chaminé $\left[{ }^{\circ} \mathrm{K}\right]$.

$T_{a}$ : temperatura do ar exterior à chaminé $\left[{ }^{\circ} \mathrm{K}\right]$.

Como última adaptação do modelo às regras de representação do 
SADE proposto, torna-se necessário criar uma expressão analítica genérica para os dois parâmetros de dispersão $\sigma y, \sigma z$. Dado que estes parâmetros dependem das classes de estabilidade atmosférica (Pasquill type - A a F) (PASQUILL, 1974) e que, para valores intermédios dessas classes (AB, B-C, etc.), os parâmetros de dispersão devem corresponder a valores médios dos obtidos para os extremos do intervalo, criou-se a seguinte expressão analítica que funciona em combinação com uma tabela que fornece seis constantes de acordo com o tipo de zona (rural ou urbana) e a classe de estabilidade (A a F) (BOUBEL et al., 1994). Essa expressão analítica é a seguinte (equação 4):

$$
\begin{aligned}
\sigma_{y} & =\frac{k_{y 1} D_{w}\left(1+k_{y 2} D_{w}\right)^{k_{y 3}}+k_{y 4} D_{w}\left(1+k_{y 5} D_{w}\right)^{k_{y 6}}}{2} \\
\sigma_{z} & =\frac{k_{z 1} D_{w}\left(1+k_{z 2} D_{w}\right)^{k_{z 3}}+k_{z 4} D_{w}\left(1+k_{z 5} D_{w}\right)^{k_{z 6}}}{2}
\end{aligned}
$$

Eq. 4. Parâmetros de dispersão atmosférica.

$k_{y 1}, \ldots, k_{y 6}$ : coeficientes de $\sigma_{y}$; tabelados em função do tipo de zona e classe de estabilidade.

$k_{z 1}, \ldots, k_{z 6}$ : coeficientes de $\sigma_{z}$; tabelados em função do tipo de zona e classe de estabilidade.

\subsection{Definição do Problema no SADE}

A introdução do modelo no SADE inicia-se com a definição de uma entidade do tipo Problema. Esta entidade define, basicamente, um domínio de variação dos parâmetros do sistema relevantes para o caso de estudo. Vamos supor que o estudo será efectuado numa região rectangular no plano $X O Y$ e que se pretende eventualmente avaliar a progressão dos poluentes até se atingir um regime permanente estável (considerou-se um período contínuo de 14400 minutos - 10 dias).

Em seguida o AD poderia definir, com base em tabelas, as funções que devolvem os coeficientes a utilizar nas expressões dos parâmetros de dispersão atmosférica. Essa operação é ilustrada na Figura 3. 


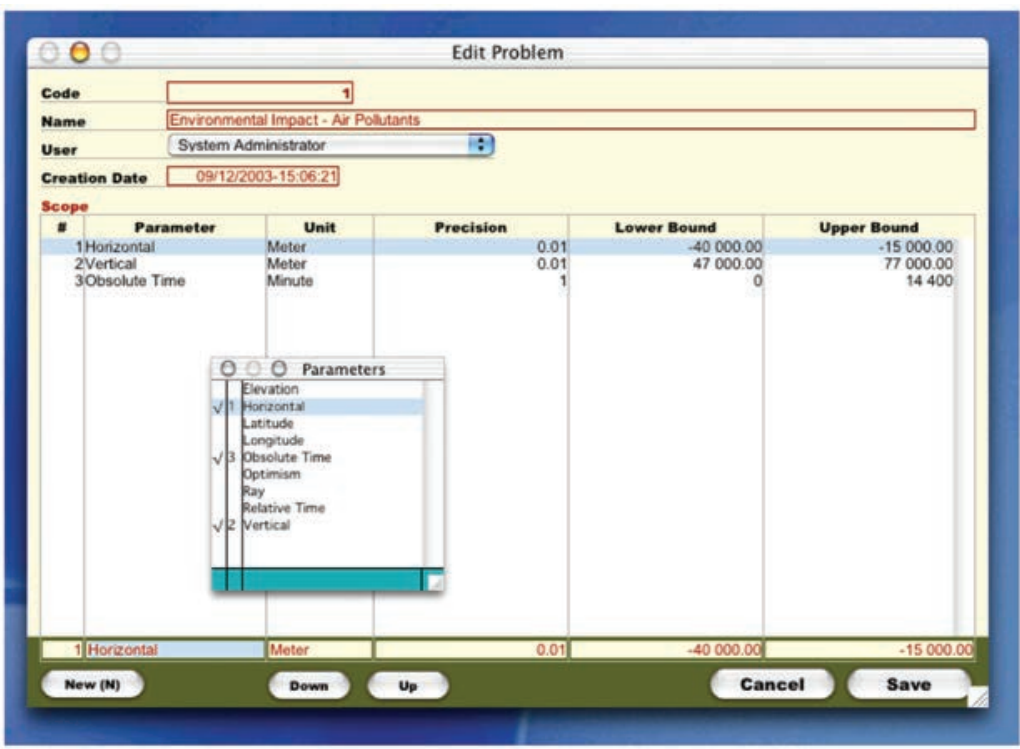

Figura 2 - Definição do Problema no SADE

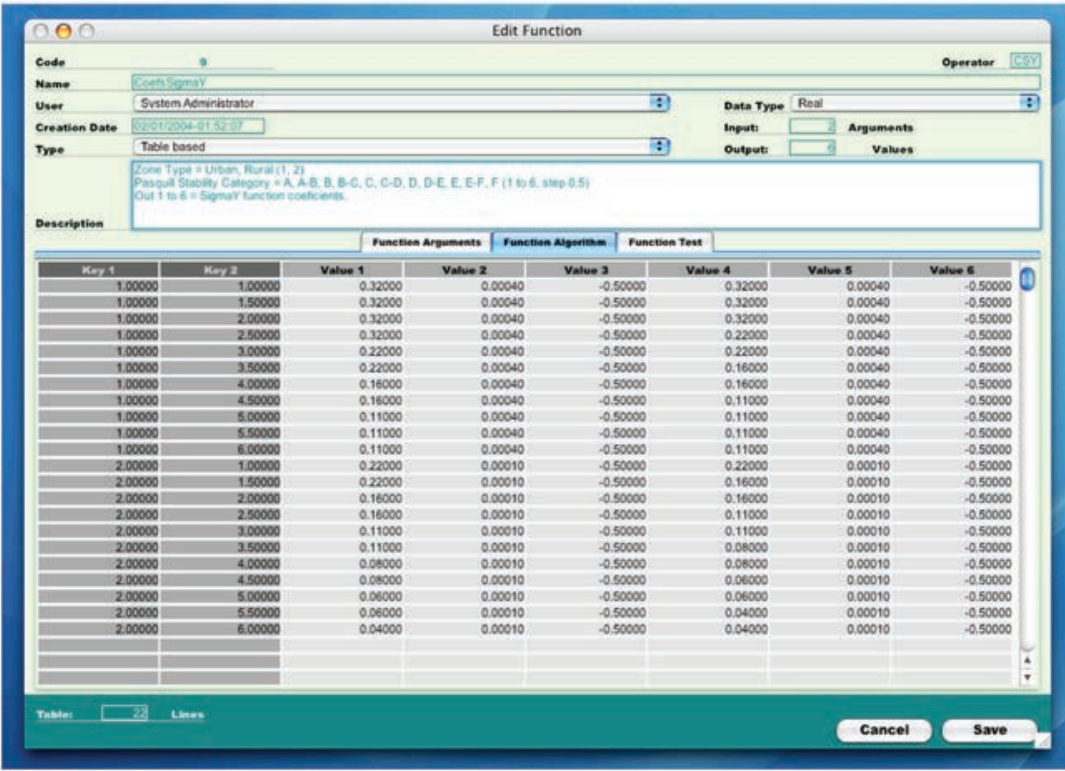

Figura 3 - Edição da função CoefsSigmaY (ecrã semelhante para CoefsSigmaZ) 
A função presente na Figura 3 (CoefsSigmaY - tabela que fornece os valores a utilizar na função que determina o parâmetro de dispersão $\sigma y$ ) é baseada na tabela apresentada na segunda página do diálogo de edição de funções. Os seis resultados devolvidos pela função correspondem aos valores presentes nas colunas Value1 a Value6, na linha que verifica a combinação de chaves Key1 Key2 dada pelos valores dos dois argumentos de entrada (Tipo de Zona e Classe de Estabilidade).

Num passo seguinte, e após ter editado uma função semelhante CoefsSigmaZ, o AD poderia definir uma nova função (agora do tipo “passo-a-passo" - step-by-step) para representar a expressão analítica da função que devolve os dois coeficientes de dispersão atmosférica $\sigma y$ e $\sigma z$ em qualquer ponto do plano. Na Figura 4, onde é apresentada a definição dos argumentos de entrada dessa função, é visível a primeira página do diálogo de edição e o cabeçalho comum a todas as três páginas.

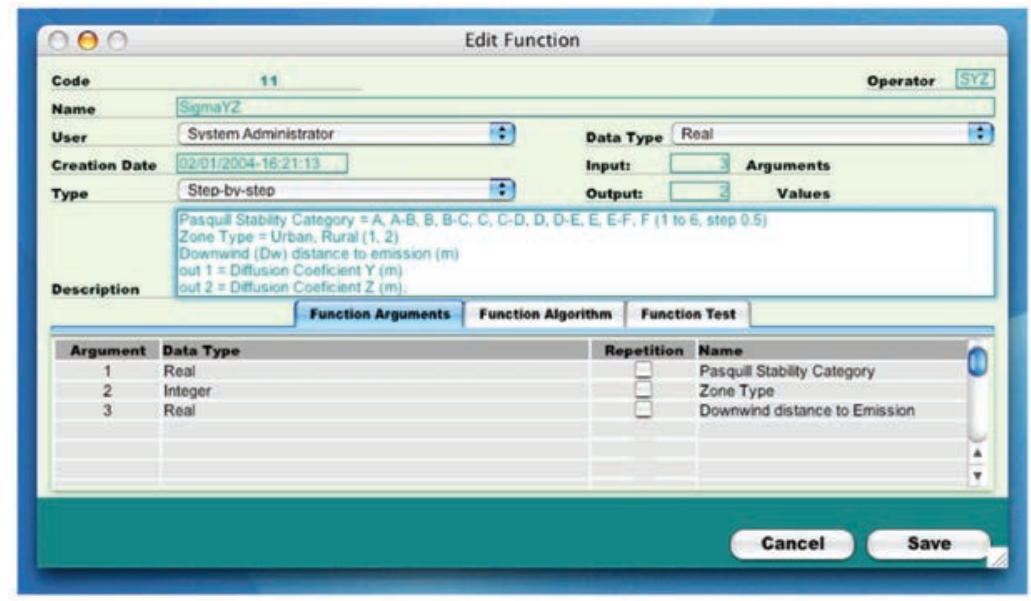

Figura 4 - Definição dos argumentos da função SigmaYZ

Na Figura 5 surge a edição do algoritmo da função cujos argumentos de entrada foram indicados na Figura 4. 


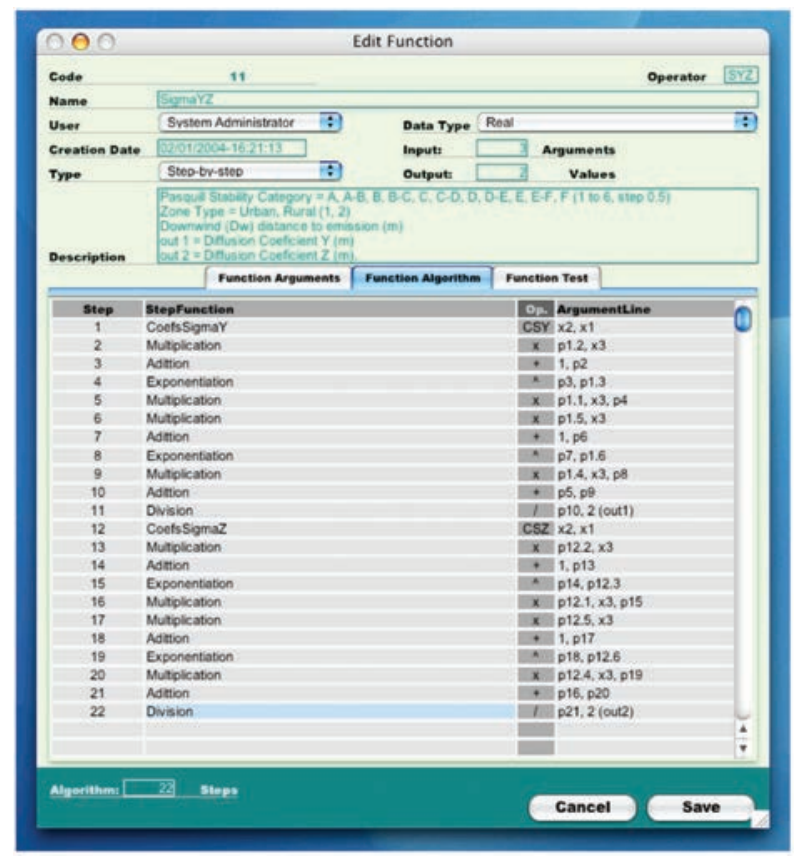

Figura 5 - Definição do algoritmo da função SigmaYZ

Através de operações semelhantes às já documentadas, o $\mathrm{AD}$ teria definido a função que calcula a elevação adicional da coluna de poluentes à saída da chaminé e estaria em condições de introduzir a expressão analítica do modelo gaussiano adaptado às regras do SADE.

O resultado único do modelo gaussiano, que representa a concentração da carga poluente provocada, por $\mathrm{m}^{3}$, no ponto de coordenadas $x_{1}, x_{2}$, $x_{3}$, corresponde ao valor devolvido pelo último passo (step 30) do algoritmo definido através do editor de expressões apresentado na Figura 6.

\subsection{Definição das Características Relevantes do Problema}

Para continuar a definição do problema de Apoio à Decisão Espacial, torna-se indispensável definir as características relevantes dos objetos envolvidos. No âmbito deste estudo ("Environmental Impact - Air Pollutants") duas categorias de objetos se evidenciam como mais importantes: 


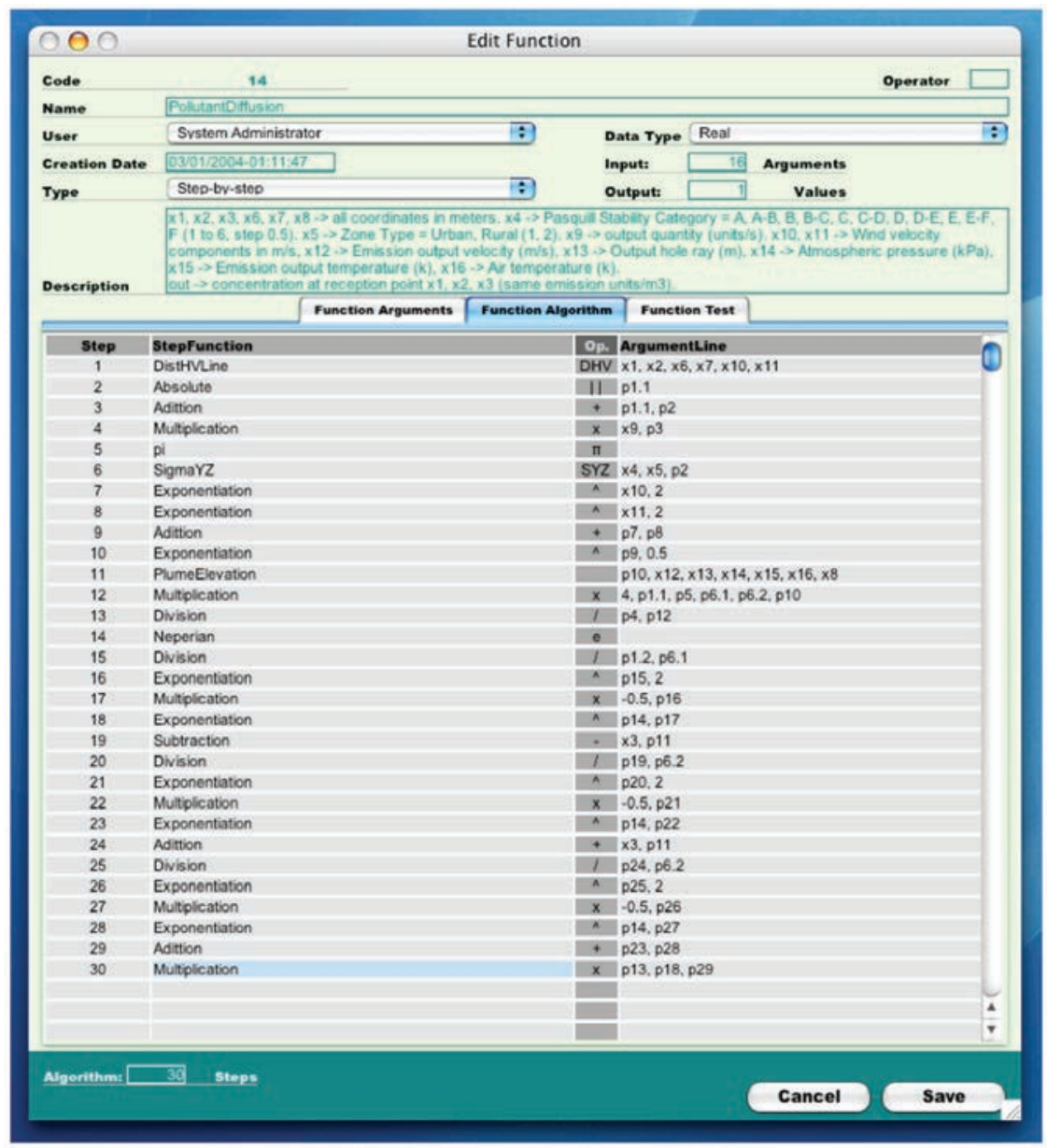

Figura 6 - Algoritmo da função PollutantDifusion

- a Subsecção Estatística, pois os estudos de impacto baseiam-se nesta unidade demográfica para obter as diferentes densidades populacionais do concelho

- o Emissor de Poluentes, pois é das suas características de emissão que dependem, em parte, as concentrações finais que vão ter impacto sobre as populações. 
Na Figura 7 é apresentado o teste da função de difusão espacial de poluentes (PolutentDifusion) criada anteriormente. É utilizando o interface espacial genérico desenvolvido para associar essa função à descrição de uma característica espacial de um objecto (chaminé) desenhado e georreferenciado na localidade de Souselas. Nesta figura é apresentada uma imagem do interface com o AD, sendo visíveis várias janelas flutuantes com diferentes tipos de elementos, codificações, operações, etc., onde o utilizador pode selecionar objetos, alterar o estado de visibilidade, desencadear operações de transformação, invocar diálogos de edição, etc. Para além dessas janelas flutuantes surge ainda a imagem representativa do problema, com os mapas de fundo e alguns objetos constituintes e uma previsão (em graduação de cores) das concentrações de poluentes ao nível do solo.

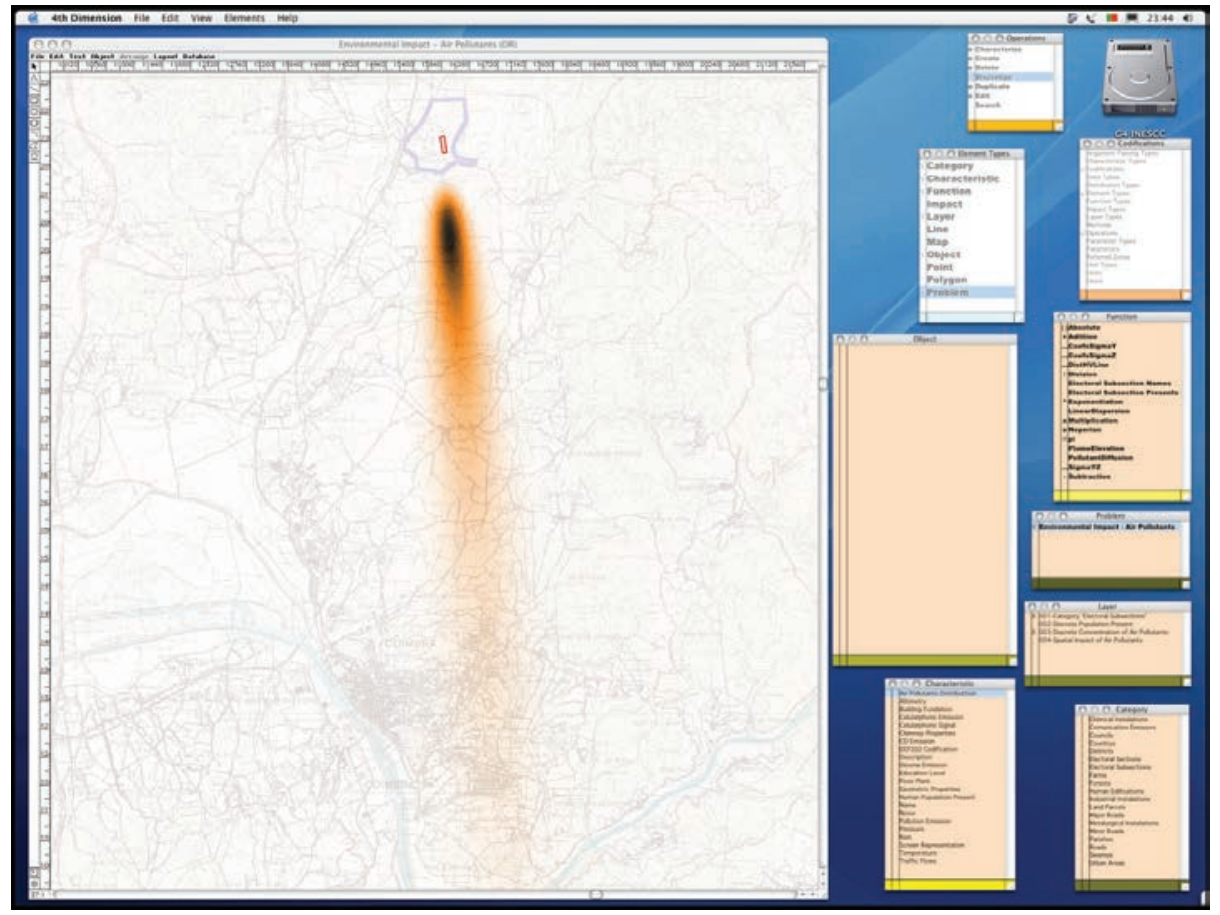

Figura 7 - Teste da função de difusão espacial de poluentes 


\section{ANÁlise ESPACIAL UTILIZANDO O SADE}

Em seguida o utilizador utiliza o interface geográfico para definir um impacto de sobreposição entre os dados relativos às concentrações de poluentes atmosféricos e aqueles relacionados com as densidades populacionais (Figura 8). Foi utilizado para tal um impacto em que a característica base (que sofre a incidência) é a relativa a densidades populacionais, e a característica incidente (neste caso apenas uma) é a relativa a concentrações de poluentes. Para o tipo de impacto desencadeado, as concentrações (associadas à característica incidente) afectam de forma 'não distributiva' a característica que sofre a incidência. Cada pessoa é afectada independentemente da densidade populacional da região onde se encontra e, como tal, a concentração não é dividida pelo número de pessoas como se fosse, por exemplo, uma distribuição de comida ou meios económicos, mas sim multiplicada.

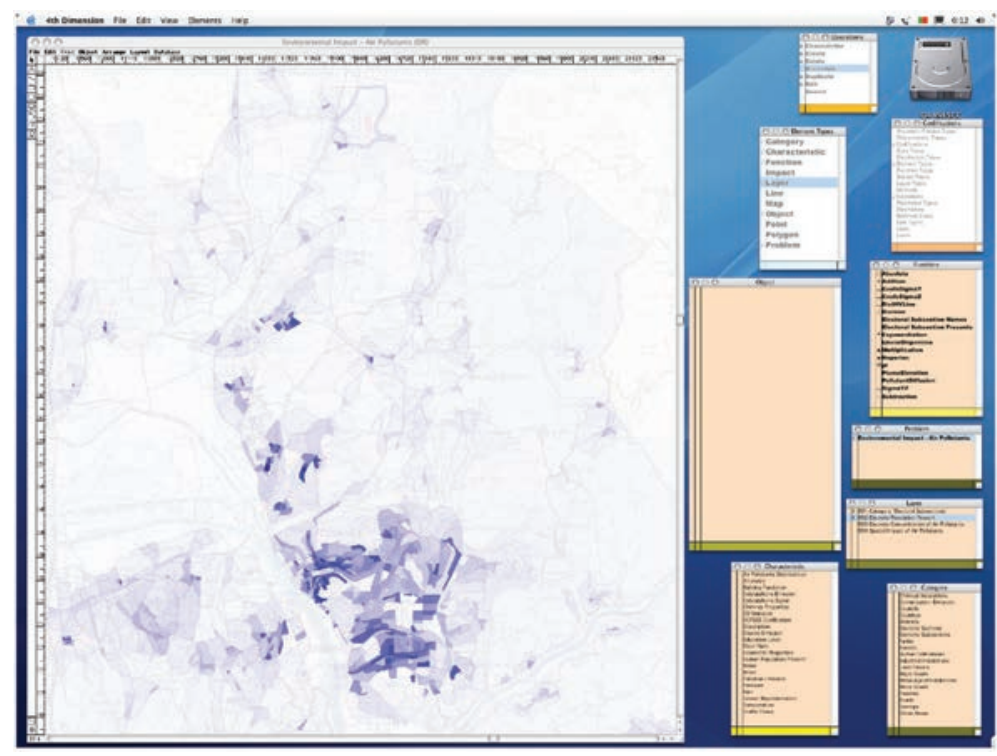

Figura 8 - Densidades Populacionais

Na Figura 9 vemos o resultado da ativação, por parte do AD, da representação geográfica do referido impacto, sendo que um tom mais escuro corresponde a maior impacto [concentração $\times$ densidade populacional]. 
Como cada impacto definido entre grupos de características produz os seus resultados associados a uma nova característica (indicada pelo AD mas manipulada internamente pelo SADE), a ativação da representação gráfica do impacto corresponde à visualização dessa nova característica.

Numa fase seguinte da exploração do problema, o AD poderia desejar obter informações mais precisas sobre o impacto gerado entre a eventual libertação de poluentes e a distribuição das populações afectadas. Para obter este efeito bastaria desencadear o método de análise espacial adequado e aplicá-lo à camada correspondente ao impacto referido. Os resultados numéricos e gráficos do método "Sobreposição Espacial Simples" são apresentados na Figura 10. Esta figura contém a mesma informação que o mapa da Figura 9, apresentada de uma forma quantitativa e legível para o utilizador, que pode assim averiguar quantas pessoas estão sujeitas a cada nível de intensidade de poluente. Nessa figura é possível verificar também que, neste método, o AD pode apenas manipular parâmetros relacionados com a visualização (mostrar, ou não, o gráfico) e não com a obtenção dos resultados em si, pois estamos na presença de um método não-compensatório.

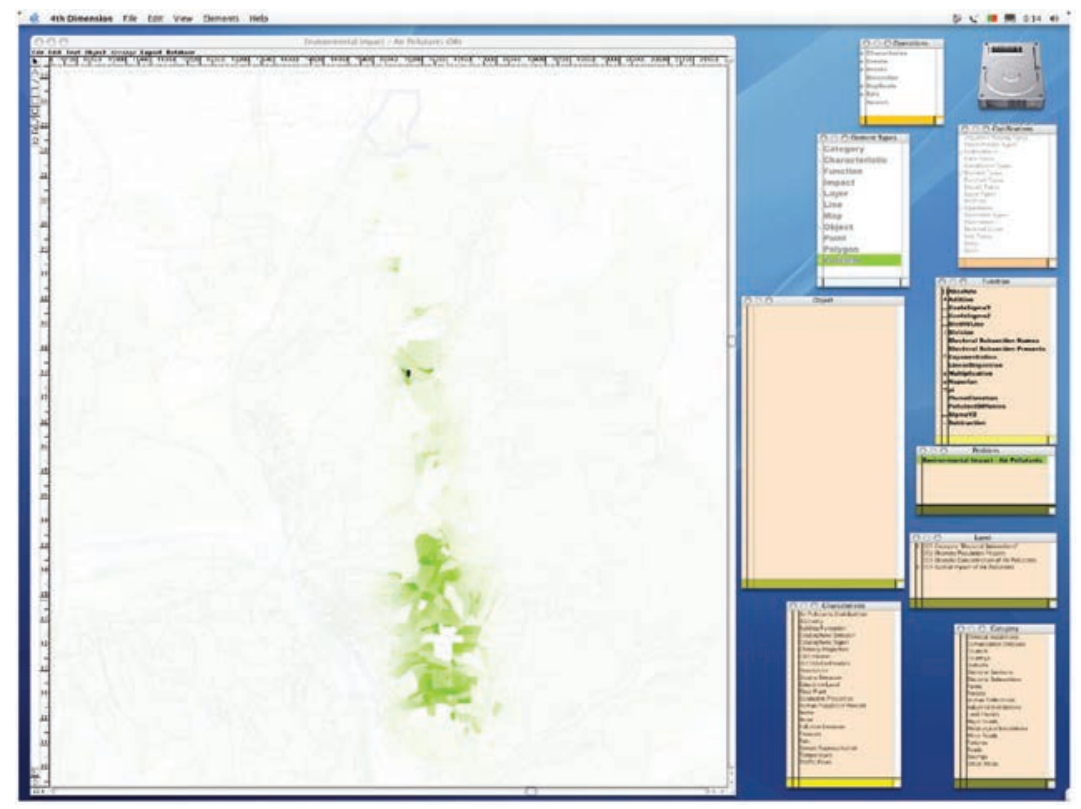

Figura 9 - Impacto de Sobreposição 


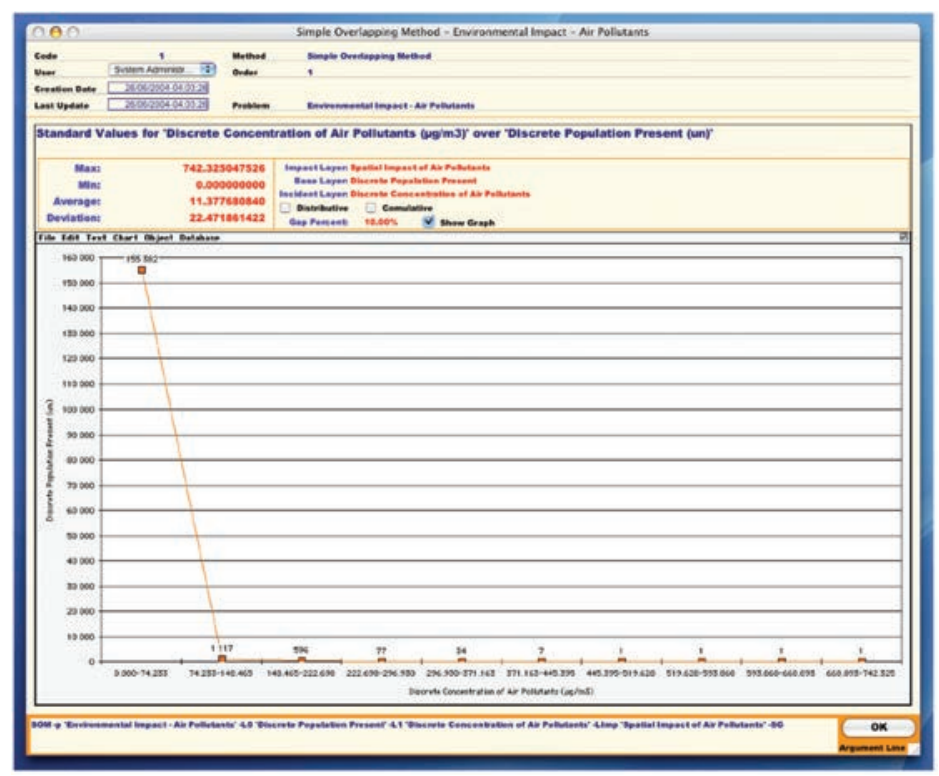

Figura 10 - Método "Sobreposição Espacial Simples”

Na Figura 11, o AD decide estudar um cenário alternativo em que identifica duas novas localizações adicionais para tratar resíduos urbanos ("Brasfemes Incinerator" em caracterização na Figura 11 e "Botão Incinerator") e procede à sua caracterização impondo valores mais reduzidos para os respectivos caudais de emissão de poluentes (reduzindo igualmente o caudal da estação inicial mas obtendo uma capacidade total de processamento superior à do primeiro cenário com uma única central). Seguidamente procede à caracterização do fenómeno da disseminação atmosférica mas fá-lo agora por categoria de objecto (e não individualmente por incineradora) para que a distribuição final traduza a influência simultânea de todas as eventuais fontes de poluentes (Figura 12). A nova situação é analisada num processo em tudo semelhante ao anterior (geração do mesmo tipo de impacto na Figura 12). Neste novo cenário a capacidade total de processamento sobe consideravelmente (cerca de 35\%). Os resultados globais, obtidos através de nova ativação do método "Sobreposição Espacial Simples" (Figura 13), traduzem também um situação de risco total ligeiramente maior para as populações humanas (média global de exposição sobe de 11.4 gg/m3 para $14.3 \mu \mathrm{g}$ / 
m3) mas a exposição máxima diminui consideravelmente (de $742.3 \mu \mathrm{g} / \mathrm{m} 3$ para $603.5 \mu \mathrm{g} / \mathrm{m} 3$ ) devido à maior dispersão espacial das incineradoras.

Este caso de estudo focou-se apenas no teste e comparação de cenários definidos pelo utilizador. Através do teste de novos cenários alternativos o AD facilmente poderia encontrar soluções de menor risco, mantendo, ou mesmo aumentando, a capacidade total de processamento de resíduos. Caso o objetivo fosse ao invés encontrar locais apropriados para as incineradoras, os dados gerados pelo SADE poderiam ser realimentados p.ex. num modelo multiobjetivo para o efeito.

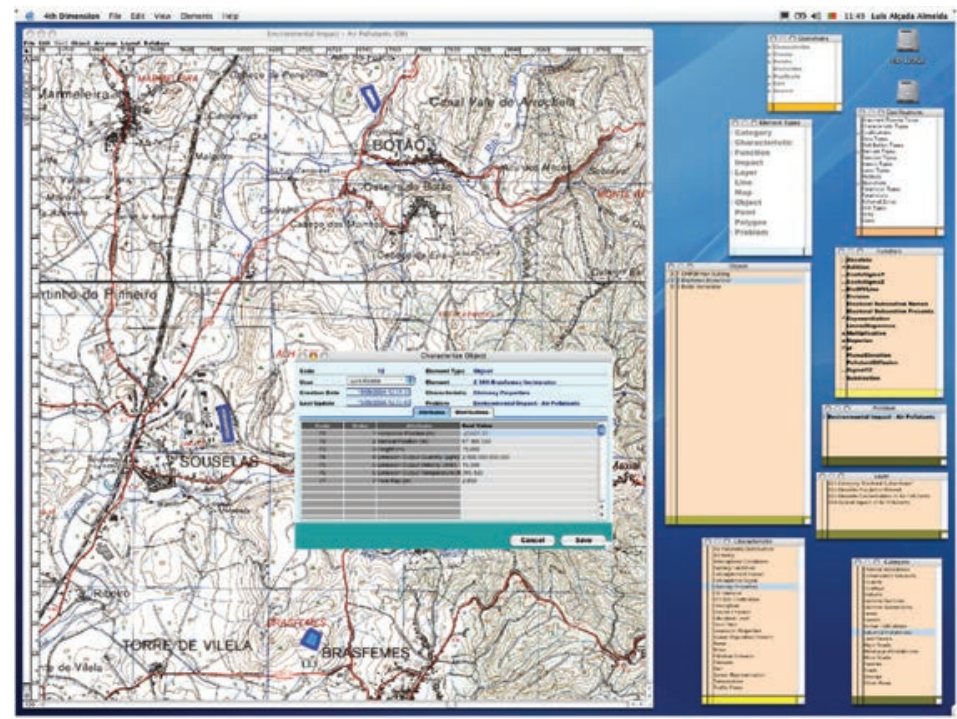

Figura 11 - Localização de duas novas Centrais de Tratamento

A utilização das capacidades analíticas do SADE proposto, operando ao nível dos atributos mono-dimensionais e da informação espacial, possibilita a produção e recolha de indicadores importantes e caracterizadores de cada um dos cenários a utilizar na construção de modelos de problemas de decisão. A natureza multidimensional do contexto que caracteriza, em geral os problemas de decisão com fortes componentes espaciais, justifica plenamente a posterior utilização de metodologias adequadas incluindo as oriundas das áreas do multiatributo e multiobjetivo. Este SADE favorece e torna mais eficaz e acessível o processo de preparação e comunicação de informação e o interface do Agente de Decisão com 
estas metodologias (CHENOWETH, 2004; NATIVIDADE-JESUS et al., 2007;

COUTINHO- RODRIGUES et al., 2011; ALÇADA-ALMEIDA et al., 2009, 2013)

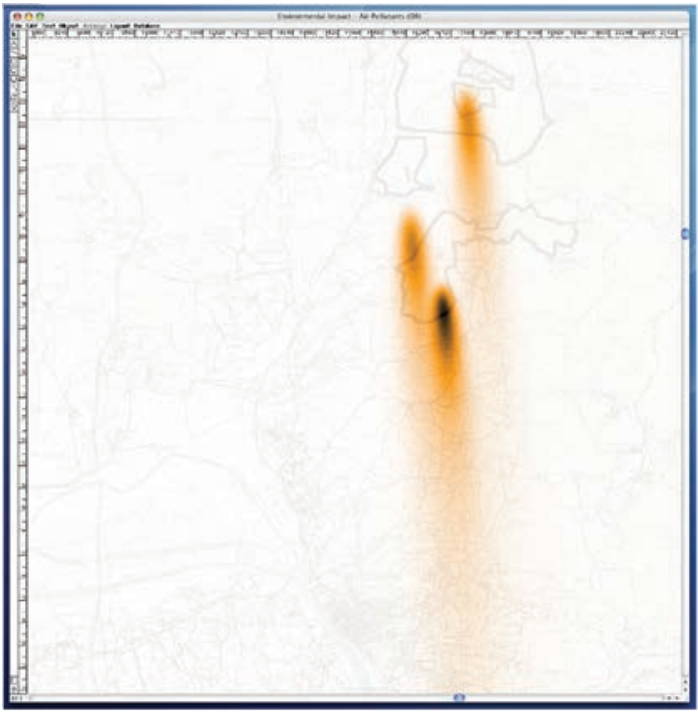

Figura 12 - Nova Distribuição de Poluentes

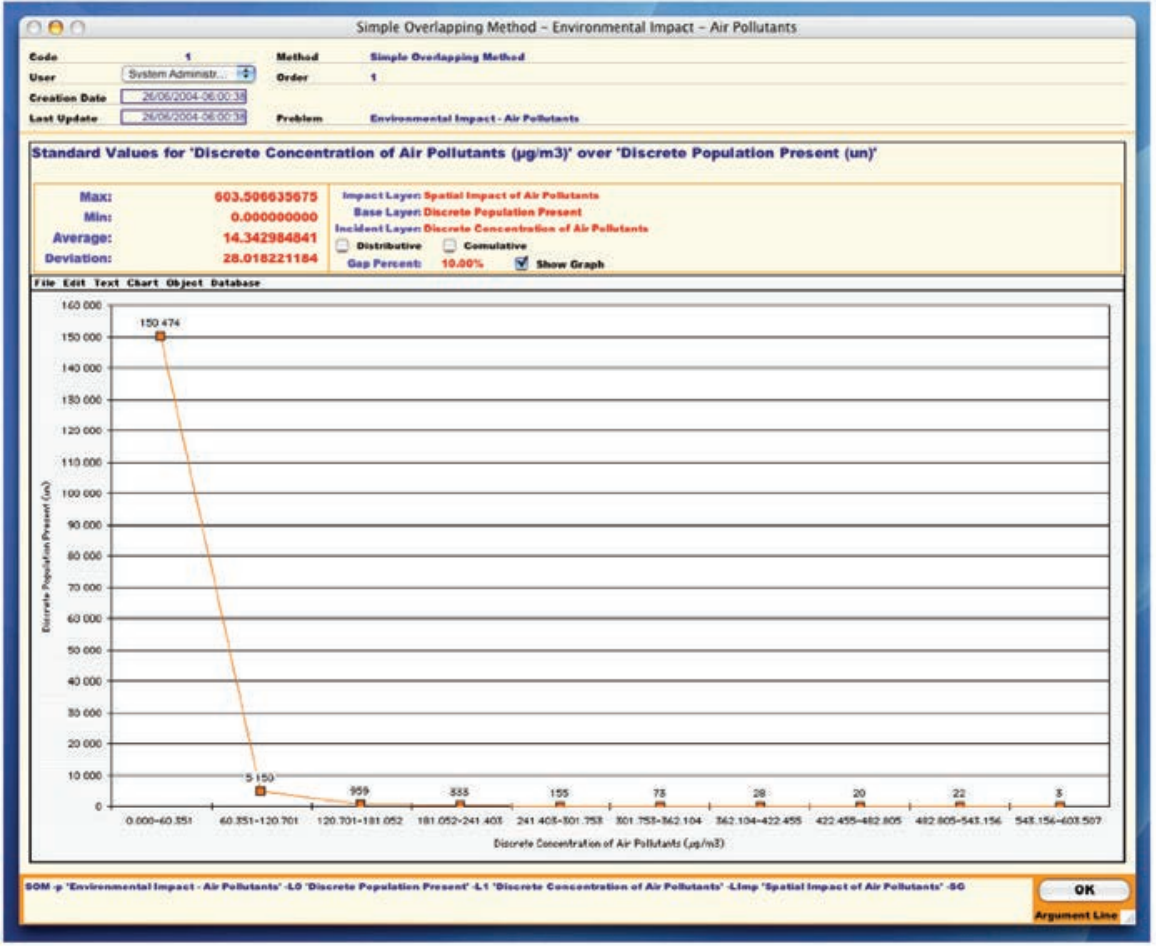

Figura 13 - Nova Aplicação do Método "Sobreposição Espacial Simples" 


\section{CONCLUSÕES}

A utilização de sistemas de informação com componentes de SIG tem vindo a assumir, na última década, uma grande relevância no apoio à decisão, quando esta se reveste de importantes componentes espaciais. O elevado nível de intuição conferido pela possibilidade de utilizar mapas interativos, tanto na entrada de dados espaciais como na análise de resultados georreferenciados (DYKES et al., 2005), contribui muito positivamente para a efetividade do processo global da tomada de decisões. Contudo, os sistemas existentes, baseados em SIG, não evoluíram no sentido de disponibilizar ao AD comum, uma plataforma genérica de modelação e análise de problemas espaciais multidimensionais. De facto, para abordar um problema concreto torna-se necessário:

- construir estruturas de dados específicas para cada problema, utilizando componentes de bases de dados mais ou menos integradas no SIG, mas de utilização inacessível para a generalidade dos AD;

- programar algoritmos de análise em linguagens nativas ou externas aos SIG, num processo que, mais uma vez, estará fora do controle do AD comum;

- conhecer a fundo as técnicas complexas de representação e tratamento de informação espacial num SIG.

Ultrapassadas estas dificuldades pelos $\mathrm{AD}$, possivelmente com o apoio e orientação de uma equipa de programadores, os sistemas assim desenvolvidos esgotam a sua utilidade com o "encerramento" do problema que lhes deu origem. De facto, as estruturas lógicas e funcionais implementadas apresentavam uma grande dependência do contexto daquele processo de decisão particular e, muitas vezes, nem os dados introduzidos são reaproveitáveis, quanto mais as estruturas e os algoritmos!

No sistema desenvolvido no âmbito deste trabalho, procuram-se endereçar processos genéricos de apoio à decisão, onde a componente evolutiva que normalmente os caracteriza possa ser abordada na sua plenitude. Assim, ao invés das estruturas de dados e algoritmos se orientarem pelo modelo de cada problema particular, é o modelo do problema que é construído utilizando as estruturas lógicas e funcionais pré- estabelecidas no sistema. Deste modo, os resultados de cada análise efectuada podem 
servir de base para localizações de novos elementos, estabelecimento de novas configurações de ligações, dimensionamento de serviços, etc. Os novos cenários que assim vão surgindo podem alimentar novos problemas de decisão (como é típico, por exemplo, nos processos de gestão urbana) que o $\mathrm{AD}$ analisará, fundamentado no conhecimento já adquirido, partindo de subconjuntos da informação gerada em anteriores processos, combinados com novos dados a introduzir e novas preferências a manifestar.

No caso de estudo apresentado a metodologia permite estudar a localização combinada de centrais de tratamento de resíduos, com vista a subsequentemente se maximizar as quantidades incineradas e minimizar o impacte, contribuindo assim para uma gestão mais eficiente e ambientalmente mais sustentável. Como argumentado, facilmente se poderá estender a outras aplicações onde seja necessária uma gestão sustentável.

\section{AgRAdecimentos}

Este trabalho foi parcialmente financiado pela Fundação Portuguesa para a Ciência e Tecnologia (FCT) sob a Ref "PEst-OE/ EEI/UI308/2014", pelo projeto Energy and Mobility for Sustainable Regions (EMSURE) - Ref ${ }^{a}$ CENTRO-07-0224-FEDER-002004, e enquadra-se na iniciativa Energy for Sustainability da Universidade de Coimbra.

\section{BIBLIOGRAFIA}

ALÇADA-ALMEIDA, L. et al. (2009) - "A Multiobjective Approach to Locate Emergency Shelters and Identify Evacuation Routes in Urban Areas". Geographical Analysis, Vol. 41, No 1, 9-29.

ALÇADA-ALMEIDA, L. et al. (2013) - "Interactive multicriteria decision support system for spatial planning analysis". Municipal Engineer, Vol. 166, $\mathrm{N}^{\circ}$ 1, 3-15.

ALMEIDA, J.P. \& COUTINHO-RODRIGUES, J. (2002) - "Estudo de Impactos Ambientais com SIG - Simulação para o Caso da Co-Incineração de Souselas”. $3^{a}$ Conferência da Associação Portuguesa de Sistemas de Informação, Coimbra, novembro 2002.

ALMEIDA, J.P. \& COUTINHO-RODRIGUES, J. (2011) - "Modelling environmental impacts over urban areas and facilities", Municipal Engineer, Vol. 164, N 2, 103-116. 
BOUBEL, R. et al. (1994) - Fundamentals of Air Pollution, 3rd ed. Academic Press, EUA. BRIGGS, G. (1969) - Plume Rise. Critical Review Series, Atomic Energy Commission, EUA.

CHENOWETH, T. et al. (2004) - "Convincing DSS Users that Complex Models are Worth the Effort". Decision Support Systems, Vol. 37, $\mathrm{N}^{\circ}$ 1, 71-82.

CORBURN, J. (2007) - "Urban land use, air toxics and public health: Assessing hazardous exposures at the neighborhood scale". Environmental Impact Assessment Review, Vol. 27, 145-160.

COUTINHO-RODRIGUES, J. et al. (2011) - "A GIS-based Multicriteria Spatial Decision Support System for Planning Urban Infrastructures.” Decision Support Systems, Vol. 51, $\mathrm{N}^{\mathrm{o}} 3,721-726$.

DYKES, J. et al. (2005) - "Exploring geovisualization", in DYKES J. et al. (eds.): Exploring Geovisualization, International Cartographic Association 1, Elsevier, Amsterdão, Países Baixos, 3-19.

GORDON, S. (1985) - Computer Models in Environmental Planning. Van Nostrand Reinhold Company, New York, 78-89.

HOLMES, N. \& MORAWSKA, L. (2006) - "A review of dispersion modelling and its application to the dispersion of particles: An overview of different dispersion models available”. Atmospheric Environment, Vol. 40, 5902-5928.

JANKOWSKI, P. et al. (1997) - "Spatial group choice: a SDSS tool for collaborative spatial decision-making", International Journal of Geographical Information Systems, Vol. 11, No 6, 577-602.

KIELY, G. (1997) - Environmental Engineering. McGraw-Hill International, Reino Unido. MAHONEY, J. (1974) - "Meteorological Aspects of Air Pollution". Industrial Pollution, Vol. 15, 409-455.

MALCZEWSKI, J. (1999) - "Spatial Multicriteria Decision Analysis". In J.-C. Thill (ed.): Spatial Multicriteria Decision Making and Analysis: a geographic information sciences approach, Ashgate Publishing Ltd., Reino Unido, 11-48.

NATIVIDADE-JESUS, E. et al. (2007) - "A Multicriteria Decision Support System for Housing Evaluation”. Decision Support Systems, Vol. 43, No 3, 779-790.

PASQUILL, F. (1974) - Atmospheric Diffusion, 2nd ed. Halstead Press, New York, EUA.

TURBAN, E. et al. (2010) - Decision support and business intelligence systems, 9th edition. Pearson Education.

USEPA (1986) - Guidelines on air quality models. US Environmental Protection Agency report EPA-450/2-78-027R.

USEPA (2003) - Revision to the Guideline on Air Quality Models: Adoption of a Preferred Long Range Transport Model and Other Revisions. Enviromental Protection Agency, Washington D.C., EUA. Disponível online no endereço 
url http://www.epa.gov/EPA-AIR/2003/April/Day-15/a8542.htm (acedido em 30 março, 2014).

ZHANG, J. et al. (2000) - "Using GIS to assess the risks of hazardous materials transport in networks". European Journal of Operational Research, Vol. 121, $\mathrm{N}^{\circ} 2$, 316- 332 . 
Série Documentos

Imprensa da Universidade de Coimbra

Coimbra University Press

2015

- U M

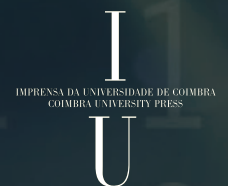

